\title{
Integrating tick density and park visitor behaviors to assess the risk of tick exposure in urban parks on Staten Island, New York
}

Erin Hassett

Cornell University

Maria Diuk-Wasser

Columbia University

Laura Harrington

Cornell University

Maria del Pilar Fernandez ( $\nabla$ pilar.fernandez@wsu.edu )

Washington State University

Research Article

Keywords: tick, risk, park, visitors, exposure, parks

Posted Date: November 8th, 2021

DOI: https://doi.org/10.21203/rs.3.rs-993154/v1

License: (c) (i) This work is licensed under a Creative Commons Attribution 4.0 International License.

Read Full License 


\section{Abstract \\ Background}

Public green spaces are important for human health, but they may expose visitors to ticks and tick-borne pathogens. We sought to understand risk and drivers of tick-preventative behavior in three popular parks on Staten Island, NY, USA by integrating tick hazard and park visitors' behaviors.

\section{Methods}

From 20 May to 19 August 2019, ticks were collected via drag cloth sampling in multiple parks, sites, and habitats to estimate tick density. Human behavior was assessed by observing usage (time spent and activity type) in each site and habitat. To evaluate risk of tick exposure, we compared park usage by demographics across sites and compared individual exposure time per site and habitat. To assess visitors' tick prevention behaviors, a knowledge, attitude, and practices (KAP) survey was administered.

\section{Results}

Three tick species (Ixodes scapularis, Amblyomma americanum and Haemaphysalis longicornis) were collected. The density of nymphs was heterogenous across parks, with the greatest density in unmaintained herbaceous habitats and trails. The fewest people entered the highest tick risk park and within-park locations. The KAP survey revealed that most respondents $(N=190)$ could not identify a nymphal stage tick when shown examples of various arthropods. Interviewees identified parks as the main location for tick exposure, but most believed they themselves had minimal risk for tick encounter. Consequently, many visitors did not conduct tick checks. People were most likely to practice tick checks if they knew multiple prevention methods and perceived a high likelihood of tick encounter.

\section{Conclusions}

By integrating acarological indices and park visitor behaviors we found a mismatch between the areas with higher tick densities and areas more frequently used by park visitors. However, this exposure risk varied among demographic groups and the type of activities. Moreover, the use of preventative measures was associated with the perceived probability of tick encounter and knowledge of tick habitat, which would modify the ultimate risk of tick encounter and disease given exposure. Our research is a first step towards identifying visitor risk, attitudes, and practices that could be targeted by optimized messaging strategies for tick bite prevention among park visitors.

\section{Introduction}


Vector-borne diseases are an increasing public health challenge in the United States, with tick-borne diseases accounting for the majority (77\%) of all cases reported in the last decade [1]. An estimated 476,000 cases of Lyme disease occur in the United States each year, predominately in the Northeast, MidAtlantic, and Upper-Midwest regions [2]. The epidemiology of Lyme disease is complex, and Borrelia burgdorferi (the major Lyme disease-causing bacterium) is maintained in enzootic transmission cycles; humans are considered incidental hosts. Thus, Lyme disease risk depends on both the density of infected ticks, typically nymphs (hereafter, tick hazard) and on human behavior which affects exposure (e.g. avoidance behaviors and use of personal protective measures) [3].

Human exposure to Ixodes scapularis ticks, the vector of B. burgdorferi in eastern United States, occurs outdoors in proximity to natural or peridomestic wooded settings. However, while links between tick density in residential yards and human disease have been extensively documented [4-10], there is little and only passive information on what percentage of infectious tick exposure comes from parks [11, 12]. Moreover, managing Lyme disease risk in peridomestic settings and natural areas differs in the fact that while humans can reduce the density of ticks by conducting environmental interventions in their yards (e.g., applying area-wide acaricides, treating rodents for ticks, or by performing landscape modifications) $[13,14]$, these interventions are not feasible at larger scales. Thus, recreational park visitors can only manage their risk by adjusting their behavior to reduce the chance of tick exposure. Commonly advised behaviors to limit tick encounters include avoiding high-risk areas by staying in the center of trails, applying tick-repellent, checking for ticks, and wearing protective clothing (i.e., permethrin-treated pants) [15]. Knowledge, attitude, and practice (KAP) surveys can identify gaps in the public's knowledge and response to tick exposure risk. Current KAP research demonstrates that respondents minimally and irregularly exercise tick preventative practices $[16,17]$. Likewise, predictors of practicing tick preventative measures vary by study and are influenced by respondents' prior experience and knowledge about and attitude towards the topic [16-18].

Estimating human-tick encounters in parks faces many challenges. Human exposure risk to ticks and pathogens has been previously estimated in public green spaces by using a drag cloth sampling technique and determining the infected tick encounter rate per hour [19] or tick encounter distance (number of meters passed until encountering a tick) on frequently used trails [20]. However, these studies only use acarological measures and do not examine human usage of the sampled areas to assess exposure. Moreover, tick density and pathogen distribution can vary widely between and within parks in the same area [21-23], resulting in spatial heterogeneities in the tick hazard. To the best of our knowledge, no previous study has simultaneously (in time and space) assessed the tick hazard and park users' behaviors that will determine the risk of human-tick encounters in parks.

This study was conducted in an urban area with high population density and extensive park systems (Staten Island, New York City), where locally acquired tick-borne diseases have been on the rise [24]. The importance of parks in dense urban areas for human well-being has been extensively studied, particularly during the COVID-19 pandemic, and includes stress reduction [25], mental fatigue relief [26, 27], violence reduction [28], and increased sense of happiness [29]. However, there is increasing concern about the 
growing health threats from tick-borne diseases in and around urban environments since green spaces, such as parks within urban centers, can provide suitable habitat for ticks infected with various tick-borne pathogens $[30,31]$.

We assessed park visitors' risk of tick encounter, combined behavioral observation assessments to determine usage of park spaces and habitats among visitors, administered KAP surveys to determine park visitors' knowledge, attitudes, and practices regarding ticks and tick-borne diseases, and sampled for ticks to determine tick densities in the areas where human data was collected. Importantly, since park visitors may be at risk for multiple tick species, we examined multiple tick vectors concurrently to better determine risk (i.e., Ixodes scapularis, Amblyomma americanum, and Haemaphysalis longicornis) [23, 32]. Integrated collection and analyses of tick and human behavioral data would allow for better tailoring of public health messaging and park management by defining areas within parks with high and low tick hazard, determining which visitors are utilizing the park areas of the highest tick hazard, and elucidating the factors influencing park visitors to engage in practicing preventative behaviors.

\section{Materials And Methods}

Field sites. Staten Island (Richmond county) is one of five boroughs in New York City (NYC), and it is the least-populated borough with an estimated population of 476,000 people as of 2018 [33,34]. The island is composed of neighborhoods exhibiting differences in housing structure and demographic and socioeconomic composition; overall, $75.2 \%$ of the population identifies as White or Caucasian, $11.7 \%$ Black or African American, 10.2\% Asian, and 18.7\% identifies as Hispanic or Latino [35]. Known as the "borough-of-parks", $18 \%$ of the total area is covered by urban parks and forests [33], and an assessment of tick populations in NYC showed that most tick species were established on Staten Island and only a few focal areas in the Bronx borough [36]. The rate of locally-acquired Lyme disease cases on Staten Island has increased from 4 to 25 per 100,000 between 2000 and 2016 [37]. Three public parks were selected on Staten Island (see Figure 1): Clove Lakes Park (40³7'07.2"N 74 $\left.06^{\prime} 41.7^{\prime \prime} \mathrm{W}\right)$, Willowbrook Park

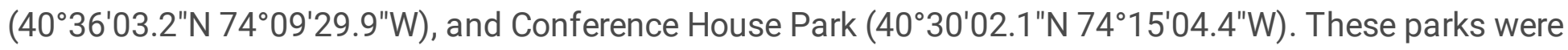
selected due to their observed high volume of park visitors and a range in tick density, with the lowest density of ticks at Clove Lakes in the north and the highest density of ticks at Conference House in the south $[23,32]$. Open spaces and hiking trails were selected in each of the parks to assess human use and tick density, and these areas were selected based on site availability in the park (smaller parks had fewer open areas and trails) and presence of potential tick habitat (Additional file 1). Prior to conducting tick sampling and human use assessments, we defined the boundaries of the open areas by determining the field of view from various points and identifying landmarks that could act as limits (i.e., paths, woodlines and waterlines). For hiking trails, we identified entry/exit points or intersections with high pedestrian traffic.

Tick collections. From 20 May to 19 August 2019, tick collections were performed once a week per site. To collect questing ticks, a $1 \mathrm{~m}^{2}$ white corduroy cloth (tick drag) was used. When sites were large enough, transects of $100 \mathrm{~m}$ were measured and drag sampled, otherwise shorter transects were drag sampled, 
and attached ticks were removed every $20 \mathrm{~m}[23,32,38]$. Ticks were placed directly in ethanol and later identified by species and sex using a Wild Heerbrugg Switzerland M5-23616 microscope and appropriate taxonomic keys [39]. Drag lengths were measured using BasicAirData GPS Logger ver. 2.2.4 app for Android and GPS Tracker Pro app for iPhone $6 \mathrm{~s}$, and lengths were verified using the program Garmin BaseCamp 4.8.3. Sites were sampled once a week between 8 AM and 7 PM, and at least three drags were performed per site. In open areas, drags were performed at the edges of the open area and within the open area (mowed lawn space). These edges often consisted of strips of vegetation along woodlines, waterlines, or natural paths. Impervious surfaces such as paved paths were excluded from sampling. For trail transects, drags were performed on the sides of the trail within the vegetation, and a $10 \mathrm{~m}$ buffer was kept between consecutive transects. Drags were not performed if vegetation was wet. Sampling sites were restricted to areas park visitors frequent to gauge risk for tick interaction (public trails or open lawn spaces, excluding inaccessible forested areas) and corresponded to the areas where human behavioral observations were performed. At Clove Lakes, two trails were unavailable for sampling after 21 July due to construction, vegetation removal, and inaccessibility (construction blockades).

Habitat classification. The habitat in each site (open space or trail) was classified into five categories: maintained grass (regularly mowed lawn), unmaintained herbaceous, leaf litter, impervious, and edge (Additional file 2). Edge habitats (i.e., strips of vegetation bordering an open area) consisted of unmaintained herbaceous or leaf litter habitats and occurred between 1) impervious and forest (i.e., strip of vegetation between paved paths at the limit of the open area and the forest), 2) maintained grass and forest (i.e., brush/natural path between maintained grass and woods), and 3) maintained grass and water (i.e., brush/natural path at the waterline). Varying numbers of drags were performed in each site type and habitat, depending on habitat availability in each park (see Additional file 3).

Unmaintained herbaceous habitats included multiflora rose (Rosa multiflora), poison ivy (Toxicodendron radicans), Japanese stiltgrass (Microstegium vimineum), jewelweed (Impatiens capensis), mugwort (Artemisia vulgaris), Japanese honeysuckle (Lonicera spp.), porcelain berry (Ampelopsis brevipedunculata), Japanese wineberry (Rubus phoenicolasius), Virginia creeper (Parthenocissus quinquefolia), smartweed (Persicaria lapathifolia), Japanese knotweed (Reynoutria japonica), blackberry (Rubus spp.), ragweed (Ambrosia spp.), goldenrod (Solidago spp.), milkweed (Asclepias syriacasedge), nettle (Urtica dioica), and wild sarsaparilla (Aralia nudicaulis). Leaf litter included oak (Quercus spp.), red oak (Quercus rubra), beech (Fagus grandifolia), maple (Acer spp.), and pine (Pinus spp.). Maintained grass habitats comprised of various graminoid species.

Park visitor observations. Park usage by visitors was determined by observing each park site for 30 min within time intervals: 9am-12pm, 12pm-3pm, 3pm-6pm (adapted from Goličnik \& Ward Thompson) [40]. With two visits per weekday and three intervals per visit, approximately 42 observations were collected for each site. In circumstances when observations for each site were incomplete (e.g. due to weather conditions), sites were observed again later in the season for the respective interval missing. For open space sites, paper maps with landmark locations were used, and the elapsed time each visitor occupied a habitat within the site was recorded in minutes (see Additional file 4). If a visitor entered and exited the 
site in under one minute, they were given an elapsed time of 0.1 minutes to reflect presence in the site. The following was recorded for each observed visitor: entrance/exit time of individual, dominant activity (e.g., walking, exercising, socializing, etc), if they were with a dog, estimated age range in 10-year intervals, and observed gender. Ages were estimated in four categories: child (0-10), teen (10-20), adult (20-60), and senior (60+). For trails, only entrance/exit time, age range, gender, and dominant activity was recorded. With this information, visitor counts (the number of unique visitors present during a specific observation session) by park, site type, and habitat were totaled and used for analysis. Furthermore, in open spaces where the activity of all individuals was visible, the mean number of minutes spent in each habitat for each age group and gender was calculated. During the process, observers did not engage with visitors to avoid influencing their behavior, and any individuals who approached observers were removed from the observational section of the study.

Knowledge, attitudes, and practices survey. We performed a semi-structured interview using a 10 min questionnaire to assess knowledge, attitudes, and practices regarding tick prevention (Additional file 5). The questionnaire comprised 27 questions related to park use, knowledge of ticks and tick-borne diseases, attitudes about perceived risk and severity, tick prevention behavior, and demographics. Questions involved a mix of open-ended, close-ended ordered, and close-ended unordered responses. Demographic and background questions included age, gender identity, race/ethnicity, highest level of education received, park visitation frequency, activities engaged in at the park, and source of information for ticks and tick-borne diseases. Knowledge questions included tick identification (Additional file 6), tick habitat, tick exposure, acquisition of the Lyme bacterium, prevention methods, and tick removal. Attitude questions included perceived severity of tick-borne diseases on Staten Island, perceived likelihood of tick encounter, reasons for not checking for ticks, and concerns about repellent use. Practice questions included frequency of repellent use, personal protection measures against ticks, and frequency of tick checks.

Participants were recruited by convenience sampling when 30 min observations were concluded so as to not interfere with the observations. Individuals who were not actively engaged in an activity (e.g. talking on the phone, running, playing sports, etc.) were approached for the survey, and all refusals and refusal reasonings were recorded. We explained the purpose of the study and only interviewed individuals over 18-years-old who gave oral consent. Individuals were able to stop the survey at any time, and the responses of only one visitor, if in a group, were recorded. Prior to administering the questionnaire, our team was trained to standardize the survey delivery and avoid biases. Prior to its implementation, the questionnaire was piloted to improve the delivery length, and we wore institutional clothing and name tag to help improve response rate.

Open-ended responses were grouped into categories and given a 0 or 1 if the response was verbalized. Similarly, close-ended unordered questions were given a 0 or 1 for verbalizing the response, and closeended ordinal questions were recorded on a Likert scale. 
Data analysis. Analyses were performed using R 2019 (R Foundation for Statistical Computing, Vienna, Austria. URL: https://www.R-project.org/) using the following packages: 'MASS' [41], 'emmeans' [42], 'MuMIn' [43], 'rms' [44], and 'car' [45].

\section{Tick density}

We evaluated the association between tick counts per $100 \mathrm{~m}^{2}$ and park identity, site type (transects in open spaces, including the edges of open spaces, or along trails), and habitat type (maintained grass, unmaintained herbaceous or leaf litter) as predictor variables. Since the sampling strategy was established to encompass the nymphal peak, the models only included unadjusted nymphal counts. Also, the nymphal season for I. scapularis ended at week 10 , so weeks 11 and 12 were removed from the analysis for this species, while for the other species, data collected over 12 weeks was considered. Because tick counts were overdispersed, a generalized linear model with a negative binomial error structure was selected for determining the variables that best predicted tick counts for each species. The length of the transect was included as an offset in the model. The models were performed separately for each tick species collected. Reference categories for park, site type, and habitat were selected based on the category with the most observations (number of drags). For I. scapularis and A. americanum models, the park reference variable was Clove Lakes, the habitat reference was unmaintained herbaceous, and site type reference was trails. For $\mathrm{H}$. longicornis models, habitat reference was unmaintained herbaceous and site type reference was trails; park was removed from the model since $H$. longicornis was only found in Conference House.

To account for model selection uncertainty, model averaging was implemented using the MuMin package by ordering competing models based on the Akaike's information criterion (AIC) value [46]. If there was more than one model with a $\triangle \mathrm{AIC}<2$ from the best ranked model, model averaging was performed [47]. Otherwise, the best ranked model was selected. Models were evaluated for multicollinearity issues by evaluating if the variance inflation factor $<4$, using rms::.vif in $\mathrm{R}$. The model coefficients were backtransformed from the log scale to determine the relative abundance of ticks per $100 \mathrm{~m}^{2}$ respective to the reference category. The mean number of predicted ticks per $100 \mathrm{~m}^{2}$ with confidence intervals was calculated for all model variables using emmeans: pairs and type= "response" in R.

\section{Park visitor observations}

Differences in visitor counts and elapsed time were compared by park, site type, habitat, and habitat exposure time was compared by gender and across age groups. Counts were modeled using a generalized linear model with a Poisson error distribution with an interaction effect between age and habitat and gender and habitat. The significance of the interactions was determined with an Analysis of Deviance (type III) table and chi-square test. Differences among interactions were analyzed using emmeans: pairwise and type= "response" with a Tukey method adjustment. To determine whether mean elapsed time spent in different habitats in open spaces differed by age group and gender, an additive linear regression model was used with the main effects of age group, gender, and habitat for each park. 
Subsequently, post-hoc pairwise comparisons between age groups and habitats were performed using emmeans: pairwise and type= "response" with a Tukey method adjustment.

\section{Risk index}

We estimated two measures of risk of encountering a nymph for all three species: the probability of encountering a nymph and the risk of exposure per person. These measures were derived from the park visitor observations and the nymphal density per park and site type, estimated from the tick density models (see Tick Density section). The probability of encountering a nymph during a 30 min period was estimated for each data point (i.e., observation). A new data point was assigned every time a person being observed moved to a different location within the area during the $30 \mathrm{~min}$ period, so one person can have one observation during that time period or several depending on their movement. We modified a passive sampling model that aims to explain the species-area relationships, in which the probability of finding a species in one area will depend on the size of the "target" area and the number of randomly distributed individuals of said species (i.e. "darts") [48]. Similarly, we can consider relative exposure time over an observation period (30 $\mathrm{min}$ ) as the "target" and tick density as "darts", thus, the probability of encountering a tick in 30 min depends on the proportion of the 30 mins spent in the observed area and tick density estimated in said observed location. We assumed that the probability of any nymph missing a person in $30 \mathrm{~min}$ in a focal point is inversely proportional to the fraction of time a person spends in the area (i.e., the longer the person stays, the greater the probability of a human-tick encounter) and the density of ticks (n):

$$
P(m i s s)=(1-t)^{n}
$$

We used the estimated tick density per $10 \mathrm{~m}^{2}$ for each site type and park, to account for variability in the tick density around the focal point where the person was observed. The estimated probability of a human-tick encounter given the proportion of time spent in each area during $30 \mathrm{~min}$ of observation and the density of ticks in each area per $10 \mathrm{~m}^{2}$, was calculated as:

$$
P(t i c k)=1-(1-t)^{n}
$$

We also estimated the risk of exposure per person per $30 \mathrm{~min}$ as:

$$
\text { Exposurerisk }=\sum_{j} T_{i} * n_{i}
$$

Where, $\mathrm{j}$ is the number of observations per person, $\mathrm{T}_{\mathrm{i}}$ is the time (in mins) elapsed for each observation and $n_{i}$ is the tick density estimated at said location.

For trails, since we were not able to record exit times in all cases, we simulated a negative binomial distribution for the time elapsed in trails using the variance from the observed data to have a more accurate measure of variability, and we used a median of $15 \mathrm{~min}$ (half of estimated period). Then, we derived the time spent in a trail as random draws from this distribution. On the other hand, if a person 
stayed during the whole observation period in the same location when observing an open area ( $T=30$ min and $t=1$ ), we forced the proportion of time $t$ as 0.99 to avoid the estimation of the probability to be independent of the tick density. The code for the risk estimate can be found at https://piliffq.github.io/tick-risk-index/.

\section{Knowledge, attitudes, and preventative practices of park visitors}

With the objective of evaluating the predictors of practicing tick prevention methods among park visitors, we focused on the predictors of performing tick checks. Tick checks can be performed by nearly every member of the public, irrespective of access or attitudes towards personal protective equipment, clothing, repellent, and checking for ticks via the CDC guidelines is the only way to guarantee that a tick has not attached, making it the single most important preventative method to practice. The KAP survey questions were organized into five sections: demographics, prior experience, knowledge, attitudes, and practices. Race/ethnicity was converted into a binomial variable (white or other) given most respondents identified as white. Education responses were grouped into "High school or less", "Some college/Associate", "Bachelors", and "Graduate". Age was categorized into six groups: 18-28, 29-39, 40-50, 51-61, 62-72, and 73-83. Questions related to prior tick experience were grouped and given a score from 0 to 4 (if yes to all, score was 4). This included whether the respondent had seen a tick before, found a tick on a pet or household member, and whether someone in the home had been diagnosed with Lyme disease. Knowledge questions were scored based on correctness, and individuals received one point per correct response. Identification knowledge score was out of sixteen points, and individuals received one point for every specimen they correctly determined was a tick and one point for every non-tick they identified correctly. Respondents received a tick habitat and bacterium acquisition score for every correct habitat they identified where ticks could be found and every correct response for how ticks can become infected with the Lyme disease bacterium. They also received a score for the total number of correct tick prevention methods they could identify, with one point for each correct method. Questions regarding knowledge and practices for tick prevention methods were open-ended. Some individuals reported practicing certain prevention methods in the practices portion of the survey but failed to report knowing about these methods in the knowledge section. In these cases, individual knowledge scores were positively adjusted to reflect practicing the behavior. Attitude questions involving perceived severity of tick-transmitted diseases on Staten Island and perceived likelihood of tick encounter were ordered on a Likert scale and scored out of five, with five being the most severe or most likely.

Generalized linear models were used to determine which variables influenced previous tick exposure, perceived severity of tick-borne diseases, and perceived probability of tick encounter. Explanatory variables included in each model (excluding the variable being modeled) were park, park visitation frequency, perceived severity of tick-borne diseases, perceived probability of tick encounter, number of prevention methods used, knowledge scores for tick identification, habitat, and prevention methods, owning a dog, education level, gender, and age group. Linear models were performed to assess factors influencing the knowledge scores for tick identification, habitat, and prevention methods with the same 
explanatory variables included as described above. Emmeans was used for post-hoc comparisons for categorical variables, and p-values were adjusted using the Tukey method. Tick check frequency was converted into a binomial variable and analyzed using a generalized linear model with a binomial regression. Model selection was conducted as described above.

\section{Results}

Association between tick density, park, site type and habitat. From 20 May to 12 August 2019, 432 drags were performed (Clove Lakes: $n=168$; Conference House: $n=134$; Willowbrook: $n=132$ ). All three habitats (maintained grass, leaf litter, and unmaintained herbaceous) were present in Clove Lakes and Willowbrook; however, no leaf litter habitat was present in Conference House sites (see Additional file 3).

A total of 10,036 ticks were collected across all parks and sites, including 7,133 $\mathrm{H}$. longicornis (adults: $n=489$; nymphs: $n=2,599$; larvae: $n=4,045), 1,972$ A. americanum (adults: $n=28 ;$ nymph: $n=157$; larvae: $\mathrm{n}=1,787$ ) and 931 l. scapularis (adults: $\mathrm{n}=0$; nymphs: $\mathrm{n}=85$; larvae: $\mathrm{n}=846$ ) (Additional file 7), with temporal variation by life stage (Additional file 8 ). Focusing on the nymphal data since we targeted the nymphal questing period, nymphs were mainly collected in transects along trails $(n=2663)$, followed by open spaces $(n=98)$, and at the edge of open spaces $(n=80)$ (Additional file 9$)$. Across habitat type, more total nymphs were collected in unmaintained herbaceous habitats $(n=2770)$, followed by maintained grass $(n=52)$, and leaf litter $(n=19)$, though counts varied by species (Additional file 10$)$.

The density (number of nymphs per $100 \mathrm{~m}^{2}$ ) of $A$. americanum was highest in Conference House and unmaintained herbaceous areas compared to maintained grass habitats (Table 1). After accounting for the drag habitat, the density of $A$. americanum nymphs in the strip of vegetation at the edge of open spaces was lower compared to trails (Table 1). For this species, the best model with the lowest AIC is presented, and no competing models were within $2 \mathrm{AIC}$. On the other hand, model averaging was conducted for $\mathrm{H}$. longicornis and I. scapularis. Model results showed that the density of I. scapularis nymphs was best explained by park and drag habitat, where the density of $I$. scapularis per $100 \mathrm{~m}^{2}$ was greatest in Conference House, in leaf litter, and in unmaintained herbaceous locations (Table 1). The density of $\mathrm{H}$. longicornis nymphs in Conference House was best described by site type and drag habitat, with densities per $100 \mathrm{~m}^{2}$ greatest in unmaintained herbaceous habitats compared to maintained grass and along trails (Table 1 ). 
Table 1

Generalized linear model (negative binomial regression) summary for each species. Open spaces had either an edge $(\mathrm{E})$ if the transect was at the limit of the area or no edge (NE) if the transect was in the interior of the open space. Relative abundance is the expected log count of nymphs for each unit of increase of the categorical variable compared to the reference variable.

$\begin{array}{llll}\text { Variable Category } & \begin{array}{l}\text { Relative abundance per } \\ 100 \mathrm{~m}^{2} ;\end{array} & \begin{array}{l}\text { p- } \\ \text { value }\end{array} & \begin{array}{l}\text { Number of predicted ticks } \\ \text { per } 100 \mathrm{~m}^{2} ;\end{array} \\ 95 \% \mathrm{Cl} & & 95 \% \mathrm{Cl}\end{array}$

A. americanum

Intercept

$0.0004(0,0.001)$

$<0.001$

Park

Clove Lakes

1

$1.13(0.33,3.83)$

Conference House

$33.09(8.12,134.95)$

$<0.001$

$37.44(17.93,78.21)$

Willowbrook

$1.69(0.36,7.83)$

0.502

$1.91(0.66,5.52)$

Site type Trail

1

$7.51(3.38,16.68)$

Open space NE

$0.87(0.23,3.36)$

0.840

$6.54(2.49,17.18)$

Open space E

$0.22(0.07,0.72)$

0.012

$1.65(0.44,6.22)$

Unmaintained

1

$8.97(3.94,20.42)$

herbaceous

Habitat

Maintained grass

$0.17(0.04,0.78)$

0.023

$1.50(0.38,5.96)$

Leaf litter

$0.67(0.14,3.13)$

0.611

$6.01(1.67,21.68)$

H. longicornis

\begin{tabular}{lllll} 
Intercept & & $0.24(0.17,0.32)$ & $<0.001$ & \\
Site type & Trails & 1 & & $762(327.1,1777)$ \\
\cline { 2 - 5 } & Open space NE & $0.43(0.09,1.84)$ & 0.258 & $380(174.2,827)$ \\
\cline { 2 - 5 } Habitat & $\begin{array}{l}\text { Open space } \mathrm{E} \\
\text { Unmaintained } \\
\text { herbaceous }\end{array}$ & $0.26(0.11,0.63)$ & 0.003 & $254(72.8,885)$ \\
\cline { 2 - 5 } & Maintained grass & $0.09(0.02,0.38)$ & 0.001 & $1435(689.1,2990)$ \\
\hline
\end{tabular}

I. scapularis

Intercept

$0.002(0,0.014)$

$<0.001$

Park

Clove Lakes

1

$7.96(0.98,64.7)$

Conference House

$4.43(1.72,10.83)$

0.006

$33.09(4.04,270.9)$

Willowbrook

$0.89(0.34,2.32)$

0.806

$7.06(0.87,57.4)$ 


\begin{tabular}{|c|c|c|c|c|}
\hline \multirow[t]{2}{*}{ Variable } & Category & $\begin{array}{l}\text { Relative abundance per } \\
100 \mathrm{~m}^{2}\end{array}$ & $\begin{array}{l}\mathrm{p}- \\
\text { value }\end{array}$ & $\begin{array}{l}\text { Number of predicted ticks } \\
\text { per } 100 \mathrm{~m}^{2} ;\end{array}$ \\
\hline & & $95 \% \mathrm{Cl}$ & & $95 \% \mathrm{Cl}$ \\
\hline \multirow[t]{3}{*}{ Site type } & Trails & 1 & & $12.5(1.67,9.41)$ \\
\hline & Open space NE & $1.15(0.35,3.84)$ & 0.927 & $12.8(1.69,97.3)$ \\
\hline & Open space E & $0.62(0.20,1.88)$ & 0.79 & $11.6(1.45,92.6)$ \\
\hline \multirow[t]{3}{*}{ Habitat } & $\begin{array}{l}\text { Unmaintained } \\
\text { herbaceous }\end{array}$ & 1 & & $31.71(4.58,219.7)$ \\
\hline & Maintained grass & $0.04(0.01,0.34)$ & 0.003 & $1.39(0.09,22.2)$ \\
\hline & Leaf litter & $1.33(0.48,3.71)$ & 0.583 & $42.25(5.46,326.9)$ \\
\hline
\end{tabular}

Park, site type, and habitat usage by park visitors. From 20 May to 19 August, a total of 5,910 individuals were observed entering the parks (Clove Lakes: $n=2,773$; Conference House: $n=1,162$; Willowbrook: $\mathrm{n}=1,975$ ). Fewer people visited Conference House compared to Clove Lakes and Willowbrook, and more people visited Clove Lakes compared to Willowbrook ( $P<0.001$ for all comparisons). Across all parks, 3,214 visitors were men and 2,632 were women $(P<0.001)$, when observed gender could be determined, and more adults visited the parks compared to children, teens, and seniors $(P<0.0001$ for all comparisons); however, adults made up the largest age range group (ages 20-60) (Table 2). More children were in Conference House compared to Clove Lakes $(P=0.0043)$ followed Willowbrook $(P=0.009)$, while more children were in Willowbrook compared to Clove Lakes $(P<0.0001)$. Teen counts were the same for all parks $(P>0.1)$. Clove Lakes had higher adult and senior counts compared to Conference House and Willowbrook ( $P<0.0001$ for all comparisons); however, more adults visited Willowbrook than Conference House $(P<0.0001)$, and Conference and Willowbrook had equal counts of seniors $(P=0.3685)$.

Regarding gender, female and male counts were higher in Clove Lakes compared to Conference House and Willowbrook parks. Counts for both genders were higher in Willowbrook compared to Conference House $(P<0.0001$ for all comparisons) (see Table 2). 
Table 2

Counts of park visitors by age group and gender. The total number (n) and percent of total visitors (\%) in each park within each group.

\begin{tabular}{|lllllll|}
\hline Park & $\begin{array}{l}\text { Gender } \\
\mathbf{n}(\%)\end{array}$ & \multicolumn{5}{l|}{$\begin{array}{l}\text { Age group } \\
\mathbf{n}(\%)\end{array}$} \\
\hline & Male & Female & Child & Teen & Adult & Senior \\
\hline Clove Lakes & 1,583 & 1,194 & 200 & 207 & 1,877 & 490 \\
& $(56.8)$ & $(43.2)$ & $(7.2)$ & $(7.4)$ & $(67.7)$ & $(17.7)$ \\
\hline Conference House & 649 & 529 & 288 & 151 & 582 & 166 \\
& $(55)$ & $(44.9)$ & $(24.3)$ & $(12.7)$ & $(49)$ & $(14)$ \\
\hline Willowbrook & 1,032 & 945 & 402 & 174 & 1,187 & 214 \\
& $(52.4)$ & $(47.6)$ & $(20.3)$ & $(8.8)$ & $(60)$ & $(10.8)$ \\
\hline
\end{tabular}

Visitor counts in each site type and habitat varied and were influenced by gender and age group in each park (see Additional file 11). Generally, open spaces were used more than trails, and impervious surfaces were used more than habitats with vegetation. In Clove Lakes, age group counts varied by habitat but not site $(P<0.0001$ and $P=0.6794$, respectively), with adults visiting impervious, impervious-maintained grass, maintained grass, and unmaintained herbaceous habitats more than other groups. Gender counts were the same across site and habitat $(P=0.0958$ and $P=0.056$, respectively). In Conference House, gender and age group counts differed by both site and habitat type $(P<0.0001$ for all comparisons), with more males visiting trails and unmaintained herbaceous habitats compared to females. Adults visited trails and open spaces most often, and adults went into impervious-maintained grass, maintained grass, and unmaintained herbaceous habitats more than other groups. In Willowbrook, age group counts differed across habitat and site type ( $P<0.0001$ for both comparisons), with adults visiting trails, open spaces, impervious, impervious-maintained grass, leaf litter, maintained grass, and unmaintained herbaceous most often. Gender counts only differed across habitat, not site $(P=0.0027$ and $P=0.7048$, respectively) with men visiting impervious, leaf litter, and maintained grass-leaf litter environments more than females.

Across all parks, visitors were exposed to maintained grass habitats for the longest time duration (Additional file 12). In Clove Lakes, exposure time to different habitats was also influenced by age but not gender ( $P<0.0001$ and $P=0.2927$, respectively) with children in the grass longer than teens, adults, and seniors ( $P<0.0001$ for all comparisons). In Conference House, exposure time varied by age and gender ( $P=0.0049$ and $P=0.002$, respectively) with children exposed longer than teens and adults $(P=0.0258$ and $P=0.0049$, respectively) and females exposed longer than men $(P=0.002)$. Age and gender did not impact habitat exposure times in Willowbrook $(P=0.2930$ and $P=0.9723$, respectively). 
Picnicking (average range across all parks: 16-25 mins), socializing (28 mins), exercising (2-29 mins), sitting (13-14 mins), tanning (8-20 mins), and engaging in arts and photography (4- 25 mins) were longstay activities that occurred in maintained grass habitats, potentially exposing many individuals to low levels of ticks, especially if occurring near a forested edge with leaf litter. Activities in more hazardous habitats such as walking (average duration across all parks: $<1-3$ mins), jogging ( $<1$ min), biking ( $<1-1$ min), and working (3 mins) were more short-stay activities that exposed fewer visitors to hazardous habitats for less time (Additional file 13).

\section{Tick hazard.}

When combining the tick density estimated at each location with the human exposure time estimated over a period of 30 min through observations, we found that both the probability of finding a tick during that period based on the density and time spent in the area was higher in trails compared to open areas for all species (see Figure 2). During a 30 min period, individuals had a small probability (median $=0.1 \%$; IQR: $0-0.2 \%$ ) of encountering an I. scapularis nymph when transiting trails, but the probability of encountering an I. scapularis nymph in open areas was almost zero (median probability: 0\% IQR: 0-0\%, $\left.\mathrm{Q}_{99}: 0.7 \%\right)$. A similar pattern was observed for A. americanum, with a median $0.02 \%$ (IQR: 0-0.08\%) probability of finding a nymph in trails and an almost zero probability of encountering them in open areas (median probability: 0\% IQR: 0-0\%, Q99: 1.4\%). For both species, the highest probability of finding a nymph was found at Conference House Park (Figure 2). H. longicornis was only found at Conference House Park, and the probability of finding a nymph was also higher in trails compared to open areas (median probability: 7.7\%, IQR: 0-15.0\% in trails and median probability: 0\%, IQR: 0-0\%, Q99: 40.3\% in open spaces). Although the probability of finding a tick in open areas was almost zero for all species, the estimated individual probabilities was highly variable and non-zero probabilities were estimated for all open spaces (Figure 2).

When we measure the risk of exposure per person during the period analyzed, we estimated an overall risk of 0.03 I. scapularis nymphs per $\mathrm{min} / \mathrm{m}^{2}$ (IQR: 0-0.06) in trails but almost zero in open spaces (0 I. scapularis nymphs per min/ $\mathrm{m}^{2}, \mathrm{IQR}: 0-0, \mathrm{Q}_{99}$ : 0.07). In comparison, we found a lower overall risk of encountering $A$. americanum (0.01 $A$. americanum nymph per min/m², IQR: 0-0.03 in trails and $0 A$. americanum nymphs per $\mathrm{min} / \mathrm{m}^{2}, \mathrm{IQR}: 0-0, \mathrm{Q}_{99}: 0.42$ in open spaces); and a higher risk of encountering $H$. longicornis (2.36 H. longicornis nymphs per $\mathrm{min} / \mathrm{m}^{2}$, IQR: 2.36-7.10 in trails, and 0 H. longicornis nymphs per min/m², IQR: 0-0, Q9g: 3.10 in open spaces). The highest risk of exposure occurred at Conference House Park for all three species.

Survey response rate, demographics, and tick experience. One hundred and ninety surveys were administered across all parks (Clove Lakes: $n=65$; Willowbrook: $n=61$, Conference House: $n=64$ ). Of the 232 park visitors who were asked to participate in the survey, 42 refused, resulting in an $18 \%$ refusal rate. Among the refusals, $52 \%$ were male, and $46 \%$ of the refusals were observed to be between $50-70$ years old. Respondents who agreed to participate were predominately residents of Staten Island $(n=176 ; 93 \%)$, 
male ( $n=109 ; 57.4 \%)$, white/Caucasian ( $n=112 ; 58.9 \%)$, and non-Hispanic/Latino ( $n=153 ; 80.5 \%)$. The age range of respondents was from 18-82 with a median age of 50 . Education ranged from having a high school degree or less $(n=41 ; 21.6 \%)$ to having a graduate degree $(n=24 ; 12.6 \%)$; however, most attended some college or received 2-year college (associates) degree $(n=71 ; 37.4 \%)$. The two main sources for acquiring information about ticks and tick-borne diseases were the internet $(n=77 ; 41.4 \%)$ and TV/radio $(n=37 ; 19.9 \%)$ (Additional file 14).

Most respondents $(60 \% ; n=114)$ had some level of past tick exposure (Additional file 14$)$. Sixty percent of visitors $(n=114)$ reported seeing a tick before. Of those who had seen a tick, 53.5\% $(n=61)$ reported finding ticks on either themselves or a household member, and $54.4 \%(n=61)$ had found ticks on a pet. Forty-four percent of respondents $(n=84)$ knew someone with Lyme disease, of which $30.9 \%(n=58)$ knew one individual and $12.4 \%(n=23)$ knew two people. Eighteen percent $(n=19)$ reported having someone in the household with Lyme disease. Prior experience with ticks was not associated with park, visitation frequency, knowledge of tick habitat, knowledge of tick phenotype, perceived probability of tick encounter, knowledge of tick prevention methods, number of prevention methods used, perceived severity, gender, age, education, or owning a dog $(P>0.05)$.

Knowledge and attitudes. Park visitors were moderately to highly knowledgeable about which specimens were ticks and which were other arthropods but less knowledgeable about disease biology (Additional file 14). Of those who claimed to have seen a tick before, most could distinguish ticks from non-ticks from a sample of eight arthropods (Additional file 6). When asked to recognize the ticks in the sample, $64 \%$ $(n=72)$ correctly identified the Dermacentor variabilis adult as a tick, while this fraction decreased to $38.1 \%(n=43)$ for $A$. americanum adults, and $26.5 \%(n=30)$ for $I$. scapularis adult. However, only $3.5 \%$ $(n=4)$ were able to tell that the I. scapularis nymph was a tick. Knowledge of tick phenotype was not affected by park, visitation frequency, knowledge of tick habitat, perceived probability of tick encounter, knowledge of tick prevention methods, number of prevention methods used, perceived severity, prior experience with ticks, gender, age, education, or owning a dog $(P>0.05)$. Additionally, most did not know how ticks become infected with the Lyme bacterium $(n=78 ; 63.7 \%)$, and $22.6 \%(n=24)$ incorrectly assumed infection was from feeding on deer. Only $8.4 \%(n=16)$ knew that infection was caused by feeding on infected mice or on infected animals in general $(8.9 \%, n=17)$.

Regarding tick habitat, most identified parks as the main source for tick exposure ( $n=82 ; 43.2 \%)$, followed by woods $(n=52 ; 27.4 \%)$ and grass $(n=32 ; 16.8 \%)$, and $12.6 \%(n=24)$ connected tick presence to the presence of deer (Additional file 14). Knowledge of tick habitat was not affected by park, visitation frequency, knowledge of tick phenotype, perceived probability of tick encounter, perceived severity of ticks, number of known prevention methods, number of used prevention methods, prior experience with ticks, education, gender, age, or owning a dog $(P>0.05)$.

There were varying responses regarding the reduction of Lyme disease on Staten Island, and visitors could respond with multiple answers (Additional file 14). Most believed that spraying pesticides $(n=63$; $34.9 \%)$, educating the public $(n=55 ; 29.6 \%)$, and reducing or controlling deer $(n=33 ; 17.7 \%)$ would control 
the disease. Fewer people reported personal protection measures $(n=15 ; 8.1 \%)$, vegetation management $(n=12 ; 6.5 \%)$, mice control $(n=6 ; 3.2 \%)$ and general tick reduction $(n=6 ; 3.2 \%)$. A small proportion $(n=19$; $10.2 \%)$ did not know what could be done, and $4.3 \%(n=8)$ said that nothing could reduce Lyme disease.

Knowledge of tick prevention measures was limited (Additional file 14); thirty-three individuals (17.4\%) did not know any prevention measures. Of the respondents who were aware of prevention methods, they knew a median of two measures. Sixty-two percent $(n=117)$ knew about insect repellent, $40 \%(n=76)$ about wearing long sleeves, $35.8 \%(n=68)$ mentioned avoiding tick habitat as a preventative measure, $24.7 \%(n=47)$ were familiar with tick checks, and $23.2 \%(n=44)$ reported knowing about tucking pants into socks or wearing long socks. Fewer people reported knowing about wearing light colored clothing to spot ticks easily $(n=15 ; 7.9 \%)$ and showering after being outdoors $(n=10 ; 5.3 \%)$. The number of known preventative measures was not influenced by park, visitation frequency, knowledge of tick phenotype, knowledge of tick habitat, perceived probability of tick encounter, perceived severity of ticks, prior experience with ticks, age, gender, or owning a dog $(P>0.05)$. However, individuals who practiced more tick prevention methods also knew more tick prevention methods $(P<0.0001)$.

Perceptions of tick-borne disease severity were moderate to high (Additional file 14). Most respondents believed tick-borne diseases were either extremely serious or very serious $(n=80 ; 42.1 \%), 15.8 \%(n=30)$ believed they were not at all serious or slightly serious, and $20 \%$ were unsure about the status of tickborne diseases on Staten Island $(n=38)$. Individuals who had a higher perceived probability of tick encounter had a higher perception of severity $(P=0.01)$. Perceived level of severity was not influenced by park, visitation frequency, knowledge of tick phenotype, knowledge of tick habitat, knowledge of tick prevention methods, number of tick prevention methods used, prior experience with ticks, age, gender, education, or owning a $\operatorname{dog}(P>0.05)$.

Practices. While most visitors attended the parks regularly, few and inconsistent personal protective measures were used to prevent tick exposure (Additional file 14). Thirty-six percent $(n=69)$ of respondents reported visiting the parks daily, $18.4 \%(n=35)$ several times a week, $10 \%(n=19)$ once a week, $10.5 \%$ $(n=20)$ once a month, $16.3 \%(n=31)$ once a year, and $6.8 \%(n=13)$ visited for the park for the first time. Walking/running ( $n=102 ; 53.7 \%)$, dog walking $(n=39 ; 20.5 \%)$, and relaxing $(n=28 ; 14.7 \%)$ were the top activities enjoyed by respondents. Respondents who practiced tick prevention methods practiced a median of two methods. Most practiced no methods (32.8\%; $n=62)$, while $30.7 \%$ practiced one $(n=58$; $30.7 \%)$ and $19 \%$ practiced two $(n=36)$ (Table 3$)$. The most used prevention methods were avoiding tick habitat $(n=58 ; 30.7 \%)$, using repellent $(n=54 ; 28.5 \%)$, wearing long sleeves $(n=49 ; 25.9 \%)$, and conducting tick checks $(n=34 ; 18 \%)$. Fewer people reported tucking pants into socks or wearing long socks $(n=26$; $13.8 \%)$, wearing light colored clothing $(n=7 ; 3.7 \%)$, or showering after being outdoors $(n=8 ; 4 \%)$. 
Table 3

The number of tick-preventative methods practiced by park visitors. This shows the count and percentage of people who practiced a certain number of methods, with most practicing none.

\begin{tabular}{|ll|}
\hline Number of methods practiced & $\begin{array}{l}\text { Number of individuals } \\
\mathbf{n}(\%)\end{array}$ \\
\hline None & $62(32.8)$ \\
\hline One & $58(30.7)$ \\
\hline Two & $36(19)$ \\
\hline Three & $20(10.6)$ \\
\hline Four & $10(5.3)$ \\
\hline Five & $2(1.1)$ \\
\hline Six & 0 \\
\hline Seven & $1(0.5)$ \\
\hline
\end{tabular}

Most people ( $n=99 ; 58 \%)$ reported checking for ticks either sometimes or always, while $42 \%(n=79)$ reported never checking for ticks (Additional file 14). Those who never checked for ticks believed they were not in an area with ticks $(24 \% ; n=46)$, they reported laziness $(12 \% ; n=23)$, forgot to check $(12 \%$, $n=22)$, never had experience with ticks $(10 \%, n=19)$, or did not think about it at the time $(10 \% ; n=19)$.

Results from the generalized linear model (Table 4) showed that tick check behavior could be best predicted by the number of tick prevention methods known, the perceived probability of tick encounter, and knowledge of tick habitat. With each unit increase in the known number of prevention methods, the odds of checking for ticks increased almost two-fold. The odds of practicing tick checks were 3.7 times more when increasing knowing no prevention methods to knowing two methods. Likewise, with each unit increase in the perceived probability of encountering a tick, the odds of checking for ticks increased 1.6 times. The odds of a park visitor checking for ticks who perceived their risk for tick encounter as "extremely likely" was 6.9 times higher than a person who perceived their risk for tick encounter as "very unlikely". Knowing tick habitat was not associated with performing tick checks. 
Table 4

An additive generalized linear regression model for predicting tick checks. The odds of checking for ticks were obtained by exponentiating the estimate.

\begin{tabular}{|lllll|}
\hline Predictor & Estimate & SE & p-value & Odds ratio \\
\hline Intercept & -0.7419 & 0.5881 & 0.2712 & NA \\
\hline Number of prevention methods known & 0.6605 & 0.2235 & 0.00312 & 1.9 \\
\hline Perceived probability of tick encounter & 0.4845 & 0.1711 & 0.00464 & 1.6 \\
\hline Knowledge of tick habitat & -0.6434 & 0.3508 & 0.06664 & 0.5 \\
\hline
\end{tabular}

\section{Discussion}

To the best of our knowledge, this is the first study to integrate the tick hazard and simultaneous assessment of human behavioral components that determine the risk of tick exposure encounters. Previous studies have examined risk for tick encounter with tick surveys [19, 20]; however, risk also depends on human usage of parks (selected areas for recreation, type of activity, and time spent) and protective behaviors to prevent tick bites and the transmission of tick-borne pathogens.

Consistent with previous studies reporting heterogenous tick densities across parks [20-23, 32], Conference House, on the southern tip of Staten Island, was the most hazardous park to encounter ticks; however, the fewest individuals visited this park, and the main tick present was $\mathrm{H}$. longicornis, which does not frequently bite humans or transmit known pathogens to humans in the United States [49-51]. This tick is a new introduction to Staten Island and may become more abundant in other Staten Island parks in the future. Trails, unmaintained herbaceous, and leaf litter habitats were the most hazardous areas for overall tick exposure. However, fewer people chose to use these areas, opting to use impervious surfaces and maintained open grass habitats, limiting their overall risk for tick exposure. The few individuals choosing to visit hazardous habitats and sites were most often males and adults. Furthermore, different types of activities may also put park visitors at risk if the activities are performed in hazardous habitats and if it exposes individuals to hazardous habitats (unmaintained herbaceous and leaf litter) for longer periods of time; however, the fewest people used these areas and the activities performed in these areas were short-stay activities. Moreover, some people, mainly children and females at Conference House, spent the most time in maintained grass, and, while tick densities were low in this habitat, there was still a minimal risk for tick exposure, primarily exposure to $\mathrm{H}$. longicornis which can be encountered readily in those maintained open grass habitats.

Our survey results indicated that respondents believed they personally had little risk for tick encounter, even though many park visitors thought tick-borne diseases were serious and exposure to ticks was mainly from parks. This perception could have influenced their tick bite risk. The likelihood of practicing tick checks was increased by visitors knowing multiple prevention methods and their perceived probability of tick encounters. Similarly, Donohoe et al. (2018) found that tick checks were predicted by having more knowledge of tick prevention and perceiving a higher risk for tick-borne disease exposure 
[52]. However, Butler et al. (2016) found that practicing tick checks was related to self-reported history of disease, and those infected with a tickborne disease were more likely to perform tick checks [53]. In our study, previous experience with ticks was not associated to increased knowledge of preventative practices. Checking the body for ticks after being outdoors (and removing ticks if found) remains one of the best ways to decrease the chances of acquiring a tick-borne infection, and public health education about ticks in parks would increase awareness and should encourage practicing multiple tick preventative behaviors, highlighting frequent tick checks when outdoors.

Limitations. Variability with the GPS tracking apps provided fluctuating levels of accuracy when tracking our dragging locations. In tracking human usage of park spaces, we did not account for unique visitor counts since it was not possible to know if the same individuals returned to the same park on different days. Thus, we were not able to adjust by heterogeneities in individual behaviors as the same individuals might have returned to the park multiple times across the summer. When administering the KAP surveys, we could not interview individuals who did not speak English, and we could only interview people who were not actively engaged in an activity. Therefore, we were not able to match individual behaviors and risk of tick encounters to their knowledge, attitudes and practices and comparisons were done qualitatively at the park level. Moreover, children and teens went into hazardous locations in the parks; however, we could not interview visitors under 18 years of age.

\section{Conclusions}

Our results from three Staten Island, NY, parks provide the first simultaneous information on tick bite risk and human behavior. Future studies that aim to understand visitor behaviors and preferences alongside real-time risk assessments could enable a refined understanding of habitat/tick control measures. In addition, these approaches could lead to more impactful visitor education, ultimately contributing to a greater overall sense of well-being and visitor confidence in local parks and the multitude of green space benefits to personal and public well-being.

\section{Abbreviations}

KAP: Knowledge, Attitudes, and Practices

\section{Declarations}

\section{ETHICS DECLARATIONS}

Protocols and procedures involving human subjects' observation and surveys were reviewed by the Institutional Review Board of Cornell University (Protocol \#: 190400878) and deemed exempt. All methods were carried out in accordance with the approved protocol and informed consent for surveys was obtained for all subjects or they legal guardians prior to participation. 
Not applicable

AVAILABILITY OF DATA AND MATERIALS

The datasets used and/or analyzed during the current study are available from the corresponding author on reasonable request.

COMPETING INTERESTS

The authors declare no competing interests.

FUNDING

This publication was supported by the Cooperative Agreement Number U01CK000509-01 between the Centers for Disease Control and Prevention and Northeast Regional Center for Excellence in Vector Borne Diseases, and the National Science Foundation's Dynamics of Integrated Socio-Environmental Systems (CNH2) program (Award \#1924061). Its contents are solely the responsibility of the authors and do not necessarily represent the official views of the Centers for Disease Control and Prevention, the Department of Health and Human Services or the National Science Foundation.

\section{CONTRIBUTIONS}

MDW and MPF conceived the study. EMH, MPF, and MDW designed the study. LCH and MDW assisted with tick sampling methodology. LCH and EMH acquired human subjects' approval. All authors created questions for the KAP survey. EMH collected the field data under LCH guidance. EMH identified ticks. EMH and MPF analyzed data. EMH wrote first draft of the paper. $\mathrm{LCH}$, MDW, and MPF contributed to writing of the paper. $\mathrm{LCH}$ provided funding for $\mathrm{EMH}$. All authors read and approved the final manuscript.

\section{ACKNOWLEDGEMENTS}

We thank all participants for taking time to share their experiences and and park managers for their interest and support of the project. We especially thank Purva Shah and Samantha Centeno for assistance during fieldwork. We would also like to thank Lynn Johnson at Cornell Statistical Consulting Unit for her advice and assistance with data analysis as well as Emily Mader for assisting with KAP survey analysis.

AUTHOR INFORMATION

Department of Microbiology and Immunology, Upstate Medical University, Syracuse, NY 14850, USA. EMH; hassette@upstate.edu.

Department of Ecology, Evolution, and Environmental Biology, Columbia University, New York, NY 10027, USA.MDW; mad2256@columbia.edu. 
Department of Entomology, Cornell University, Ithaca, NY 14850, USA.

LCH; Ich27@cornell.edu.

Paul G. Allen School for Global Health, Washington State University, Pullman, WA 99164 USA. MPF;

Email: pilar.fernandez@wsu.edu.

CORRESPONDING AUTHOR

Correspondence to Maria del Pilar Fernandez.

\section{References}

1. Rosenberg R, Lindsey NP, Fischer M, Gregory CJ, Hinckley AF, Mead PS, et al. Vital Signs: Trends in Reported Vectorborne Disease Cases - United States and Territories, 2004-2016. Morb Mortal Wkly Rep. 2018;67:496-501.

2. Centers for Disease Control and Prevention. Data and surveillance. 2019.

https://www.cdc.gov/lyme/datasurveillance/index.html?

CDC_AA_refVal=https\%3A\%2F\%2Fwww.cdc.gov\%2Flyme\%2Fstats\%2Findex.html. Accessed 25 Oct 2019.

3. Diuk-Wasser MA, VanAcker MC, Fernandez MP. Impact of Land Use Changes and Habitat Fragmentation on the Eco-epidemiology of Tick-Borne Diseases. J Med Entomol. 2020.

4. Cromley EK, Cartter ML, Mrozinski RD, Ertel S-H. Residential setting as a risk factor for Lyme disease in a hyperendemic region. American Journal of Epidemiology. 1998;147:472-7.

5. Dister SW, Fish D, Denise SMB, Frank H, Wood AL. Landscape characterization of peridomestic risk for Lyme disease using satellite imagery. 1997.

6. Connally NP, Ginsberg HS, Mather TN. Assessing peridomestic entomological factors as predictors for Lyme disease. Journal of Vector Ecology. 2006;31:364-70.

7. Mead P, Hook S, Niesobecki S, Ray J, Meek J, Delorey M, et al. Risk factors for tick exposure in suburban settings in the northeastern United States. Ticks and Tick-borne Diseases. 2018;9:319-24.

8. Finch C, Salim Al-Damluji M, Krause P, Niccolai L, Steeves T, O’Keefe C, et al. Integrated Assessment of Behavioral and Environmental Risk Factors for Lyme Disease Infection on Block Island, Rhode Island. PloS one. 2014;9:e84758.

9. Fischhoff IR, Bowden SE, Keesing F, Ostfeld RS. Systematic review and meta-analysis of tick-borne disease risk factors in residential yards, neighborhoods, and beyond. BMC Infect Dis. 2019;19:1-11.

10. Hahn MB, Bjork JKH, Neitzel DF, Dorr FM, Whitemarsh T, Boegler KA, et al. Evaluating acarological risk for exposure to Ixodes scapularis and Ixodes scapularis -borne pathogens in recreational and residential settings in Washington County, Minnesota. Ticks and Tick-borne Diseases. 2018;9:340-8.

11. Falco R, Fish D. Ticks parasitizing humans in a Lyme disease endemic area of southern New York State. American Journal of Epidemiology. 1988;128:1146-52. 
12. Jordan RA, Egizi A. The growing importance of lone star ticks in a Lyme disease endemic county: Passive tick surveillance in Monmouth County, NJ, 2006 - 2016. PLOS ONE. 2019;14:e0211778.

13. Connally NP, Ginsberg HS, Mather TN. Assessing peridomestic entomological factors as predictors for Lyme disease. J Vector Ecol. 2006;31:364-70.

14. Hinckley AF, Meek JI, Ray JAE, Niesobecki SA, Connally NP, Feldman KA, et al. Effectiveness of residential acaricides to prevent Lyme and other tick-borne diseases in humans. The Journal of Infectious Diseases. 2016;214:182-8.

15. Centers for Disease Control and Prevention. Preventing tick bites on people. Centers for Disease Control and Prevention. 2020. https://www.cdc.gov/ticks/avoid/on_people.html. Accessed 28 Jul 2021.

16. Shadick NA, Daltroy LH, Phillips CB, Liang US, Liang MH. Determinants of tick-avoidance behaviors in an endemic area for Lyme disease. American Journal of Preventive Medicine. 1997;13:265-70.

17. Bayles BR, Evans G, Allan BF. Knowledge and prevention of tick-borne diseases vary across an urbanto-rural human land-use gradient. Ticks and Tick-borne Diseases. 2013.

18. Donohoe H, Omodior O, Roe J. Tick-borne disease occupational risks and behaviors of Florida Fish, Wildlife, and Parks Service employees - A health belief model perspective. 2018.

19. Han GS, Stromdahl EY, Wong D, Weltman AC. Exposure to Borrelia burgdorferi and Other Tick-Borne Pathogens in Gettysburg National Military Park, South-Central Pennsylvania, 2009. Vector-Borne and Zoonotic Diseases. 2014;14:227-33.

20. Falco RC, Fish D. Potential for exposure to tick bites in recreational parks in a Lyme disease endemic area. American Journal of Public Health. 1989;79:12-5.

21. Johnson T, Graham C, Boegler K, Cherry C, Maes S, Pilgard M, et al. Prevalence and diversity of tickborne pathogens in nymphal Ixodes scapularis (Acari: Ixodidae) in Eastern National Parks. Journal of Medical Entomology. 2017;54:742-51.

22. Simmons AE, Manges AB, Bharathan T, Tepe SL, McBride SE, DiLeonardo MQ, et al. Lyme Disease Risk of Exposure to Blacklegged Ticks (Acari: Ixodidae) Infected with Borrelia burgdorferi (Spirochaetales: Spirochaetaceae) in Pittsburgh Regional Parks. Journal of Medical Entomology. 2019.

23. VanAcker MC, Little EAH, Molaei G, Bajwa WI, Diuk-Wasser MA. Enhancement of risk for lyme disease by landscape connectivity, New York, New York, USA. Emerging Infectious Diseases. 2019;25:113643.

24. New York City Department of Health and Mental Hygiene. NYC Interactive Health Data System Communicable Disease Surveillance Data. EpiQuery. 2018. https://a816health.nyc.gov/hdi/epiquery/. Accessed 3 Jun 2021.

25. Ward Thompson C, Roe J, Aspinall P, Mitchell R, Clow A, Miller D. More green space is linked to less stress in deprived communities: Evidence from salivary cortisol patterns. Landscape and Urban Planning. 2012;105:221-9. 
26. Hartig T, Evans GW, Jamner LD, Davis DS, Gärling T. Tracking restoration in natural and urban field settings. Journal of Environmental Psychology. 2003;23:109-23.

27. Kaplan S. The restorative benefits of nature: Toward an integrative framework. Journal of Environmental Psychology. 1995;15:169-82.

28. Garvin EC, Cannuscio CC, Branas CC. Greening vacant lots to reduce violent crime: A randomised controlled trial. Injury Prevention. 2013;19:198-203.

29. Yulistia Rahayu V, Frinaldi A, Khaidir A. The influence of green open space and tourism-conscious work culture on the happiness of the people in Solok Regency. In: IOP Conference Series: Earth and Environmental Science. Institute of Physics Publishing; 2019.

30. Mathews-Martin L, Namèche M, Vourc'h G, Gasser S, Lebert I, Poux V, et al. Questing tick abundance in urban and peri-urban parks in the French city of Lyon. Parasites Vectors. 2020;13:1-9.

31. Bellato A, Pintore MD, Catelan D, Pautasso A, Torina A, Rizzo F, et al. Risk of tick-borne zoonoses in urban green areas: A case study from Turin, northwestern Italy. Urban Forestry \& Urban Greening. 2021;64:127297.

32. Tufts DM, VanAcker MC, Fernandez MP, DeNicola A, Egizi A, Diuk-Wasser MA. Distribution, hostseeking phenology, and host and habitat associations of Haemaphysalis longicornis ticks, Staten Island, New York, USA. Emerging infectious diseases. 2019;25:792-6.

33. Kulasekera VL, Kramer L, Nasci RS, Mostashari F, Cherry B, Trock SC, et al. West Nile virus infection in mosquitoes, birds, horses, and humans, Staten Island, New York, 2000. Emerging Infectious Diseases. 2001;7:722-5.

34. U.S. Census Bureau. 2010 Census data. 2010. https://www.census.gov/population/www/cen2010/glance/. Accessed 1 Jan 2018.

35. U.S. Census Bureau QuickFacts: Richmond County (Staten Island Borough), New York. https://www.census.gov/quickfacts/richmondcountystatenislandboroughnewyork. Accessed 29 Oct 2019.

36. Barbot O. 2019 DOHMH Advisory \#12: Tick-borne Disease Advisory. New York City Department of Health and Mental Hygiene.

37. New York City Department of Health and Mental Hygiene. Epiquery: NYC Interactive Health Data System - Communicable Disease Surveillance Data. 2018. https://nyc.gov/health/epiquery.

38. Rulison EL, Kuczaj I, Pang G, Hickling GJ, Tsao JI, Ginsberg HS. Flagging versus dragging as sampling methods for nymphal Ixodes scapularis (Acari: Ixodidae). Journal of Vector Ecology. 2013;38:163-7.

39. Keirans JE, Litwak TR. Pictorial key to the adults of hard ticks, family Ixodidae (Ixodida: Ixodoidea), east of the Mississippi River. Journal of medical entomology. 1989;26:435-48.

40. Goličnik B, Ward Thompson C. Emerging relationships between design and use of urban park spaces. Landscape and Urban Planning. 2010. 
41. Ripley BR, Venables B, Bates DM, Hornik K, Gebhardt A, Firth D. Package "MASS": Support functions and datasets for Venables and Ripley's MASS. 2019.

42. Lenth R, Singmann H, Love J, Buerkner P, Herve M. Package "emmeans": estimated marginal means, aka least-squares means. 2020.

43. Bartoń K. Package “MuMIn”: multi-model inference. 2019.

44. Harrell FE. Package "rms" title regression modeling strategies. 2020.

45. Fox J, Weisberg S, Price B, Adler D, Bates D, Baud-Bovy G, et al. Package "car": companion to applied regression. 2020.

46. Burnham KP, Anderson DR. Model selection and multimodel inference: a practical informationtheoretic approach. 2nd edition. New York: Springer; 2002.

47. Symonds MRE, Moussalli A. A brief guide to model selection, multimodel inference and model averaging in behavioural ecology using Akaike's information criterion. Behav Ecol Sociobiol. 2011;65:13-21.

48. Nicholas J. Gotelli, Gary Graves. Ch. 8: Species-Area Relationships. In: Null Models in Ecology. Washington D.C.: Smithsonian Institution Press; 1996. https://www.uvm.edu/ ngotelli/nullmodelspage.html. Accessed 14 Jul 2021.

49. Tufts DM, Goodman LB, Benedict MC, Davis AD, VanAcker MC, Diuk-Wasser M. Association of the invasive Haemaphysalis longicornis tick with vertebrate hosts, other native tick vectors, and tickborne pathogens in New York City. bioRxiv. 2020;:2020.07.01.182626.

50. Beard CB, Occi J, Bonilla DL, Egizi AM, Fonseca DM, Mertins JW, et al. Multistate Infestation with the Exotic Disease-Vector Tick Haemaphysalis longicornis - United States, August 2017-September 2018. MMWR Morb Mortal Wkly Rep. 2018;67:1310-3.

51. Wormser GP, McKenna D, Piedmonte N, Vinci V, Egizi AM, Backenson B, et al. First Recognized Human Bite in the United States by the Asian Longhorned Tick, Haemaphysalis Iongicornis. Clinical Infectious Diseases. 2020;70:314-6.

52. Donohoe H, Omodior O, Roe J. Tick-borne disease occupational risks and behaviors of Florida Fish, Wildlife, and Parks Service employees - A health belief model perspective. Journal of Outdoor Recreation and Tourism. 2018;22:9-17.

53. Butler AD, Sedghi T, Petrini JR, Ahmadi R. Tick-borne disease preventive practices and perceptions in an endemic area. Ticks and Tick-borne Diseases. 2016;7:331-7.

\section{Figures}




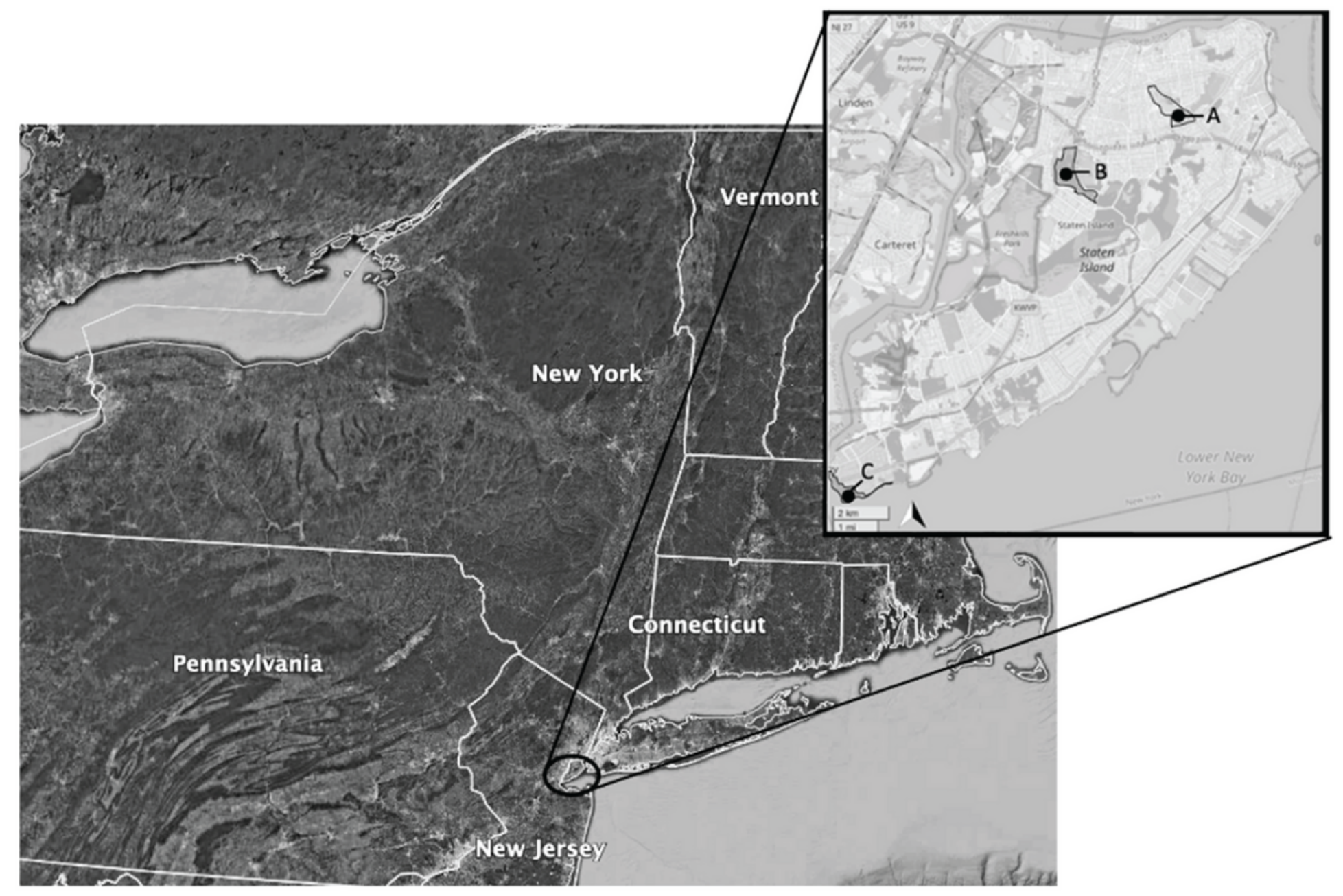

Figure 1

Park locations studied on Staten Island. A: Clove Lakes; B: Willowbrook; C: Conference House. 
Probability of tick encounter

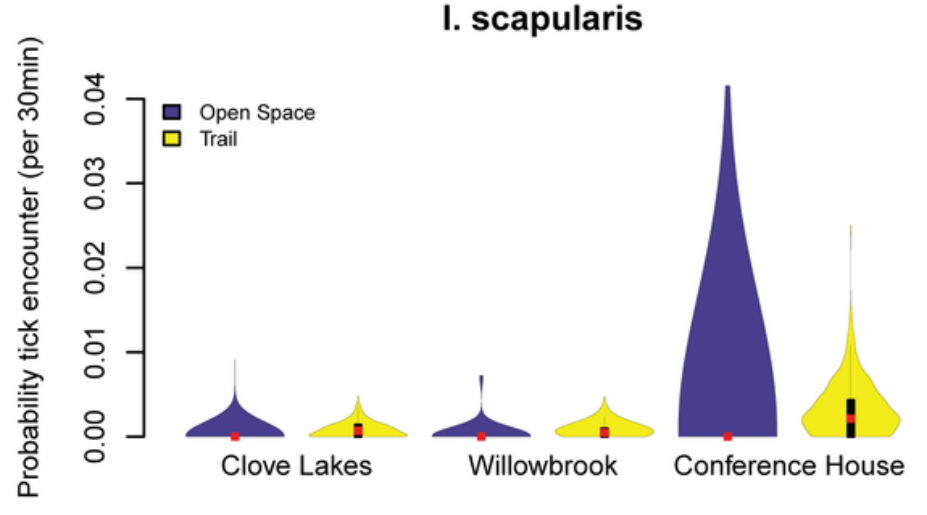

A. americanum

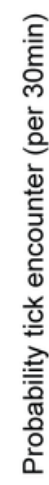
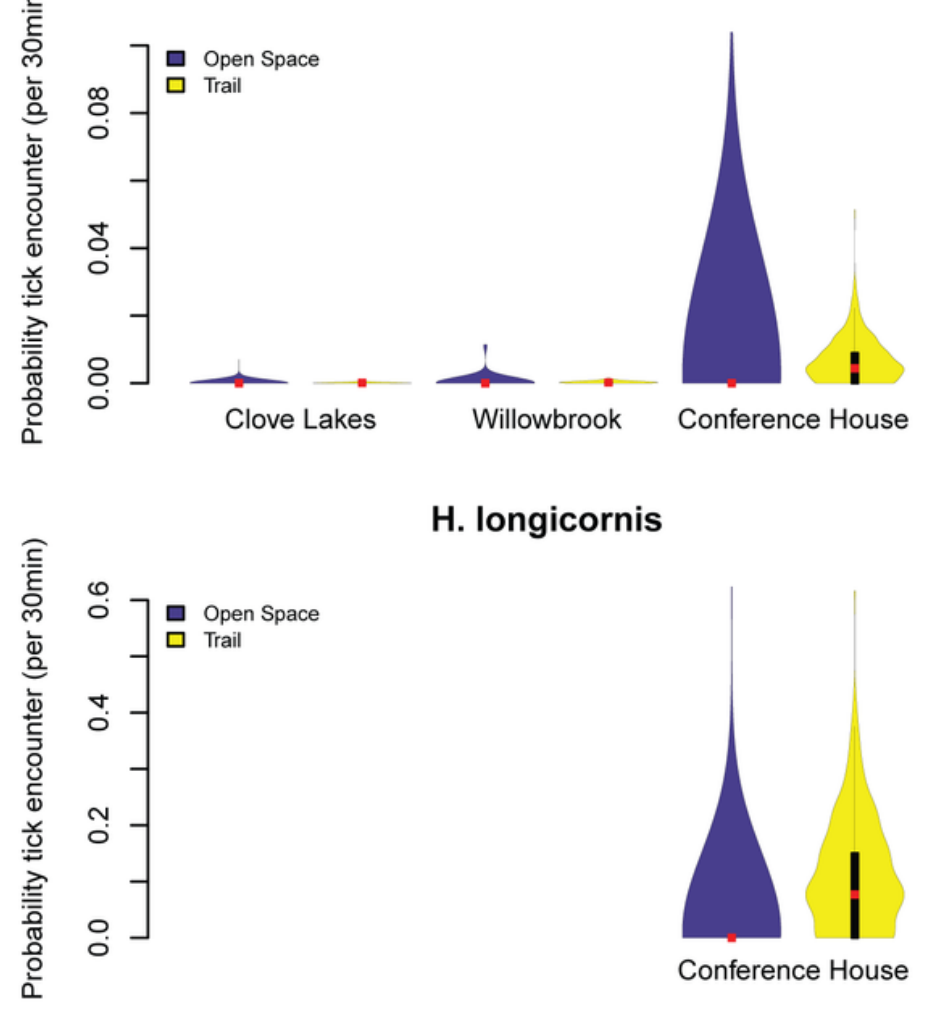

H. longicornis

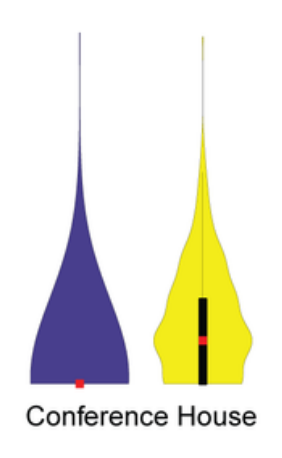

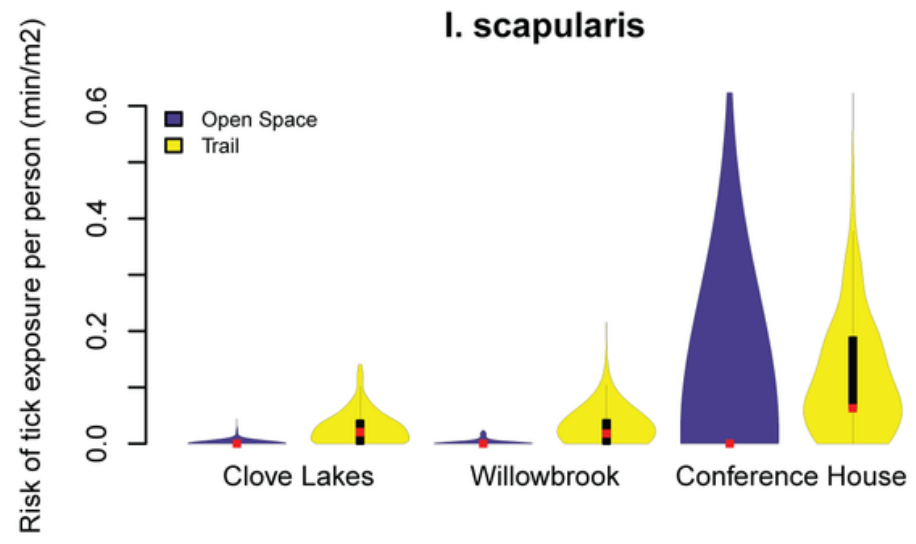

Risk of exposure per person

A. americanum

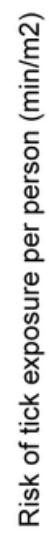
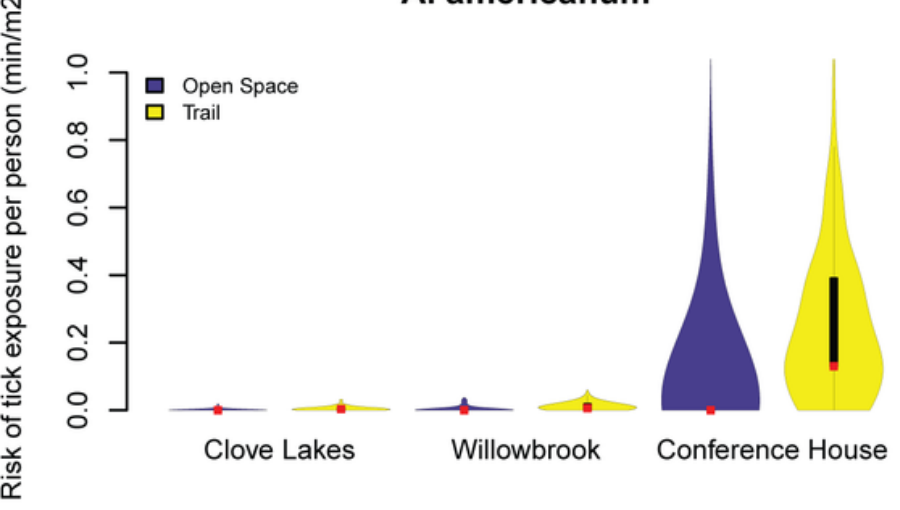

H. longicornis

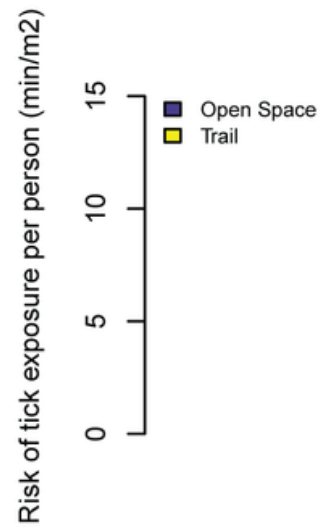

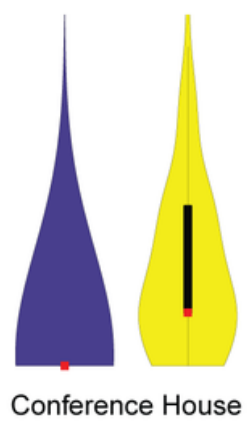

Figure 2

The probability of tick encounter and risk of tick exposure per person across park and site type.

\section{Supplementary Files}

This is a list of supplementary files associated with this preprint. Click to download.

- Additionalfile1.pdf

- Additionalfile2.pdf 
- Additionalfile3.pdf

- Additionalfile4.pdf

- Additionalfile5.pdf

- Additionalfile6.pdf

- Additionalfile7.pdf

- Additionalfile8.pdf

- Additionalfile9.pdf

- Additionalfile10.pdf

- Additionalfile11.pdf

- Additionalfile12.pdf

- Additionalfile13.pdf

- Additionalfile14.pdf 